\title{
MAPPING OF QUANTITATIVE TRAIT LOCI FOR EXSERTED STIGMA RATE BY USING (ZS97B/MH63) RIL POPULATION
}

\author{
Jiping Tong*, Zhengshu Han and Aonan Han \\ Crop Research Institute, Academy of Agricultural Sciences, Tianjin-300384, China
}

Keywords: Rate of exserted stigma, QTL mapping, Recombinant inbred lines, Rice (Oryza sativa L.)

\begin{abstract}
For increasing pollination and seed set, stigma exsertion has been identified as a major component in hybrid rice. By using a recombinant inbred line (RIL) population derived from an important Indica rice cross between Zhenshan97 and Minghui63, a molecular marker-based analysis of quantitative trait loci (QTL) for stigma exsertion was performed. As a result, six prominent QTL were detected for the exserted stigma rate on chromosome 6, 2, 9, 3, 5 and 1, respectively. qSER-1-1, qSER-2-1, qSER-5-1, qSER-6-1, qSER-15-1, and qSER-18-1 explained 8.1515, 4.6657, 7.2387, 4.8997, 6.925 and $6.9291 \%$ of the total phenotypic variance, respectively. In addition, for the $q S E R-1-1$ and $q S E R-2-1$, the ZS97B allele increased exserted stigma rate by about 4.3484 and 3.2836\%, respectively; while for $q S E R-5-1, q S E R-6-1, q S E R-15-1$ and $q S E R-18-1$, the MH63 allele increased exserted stigma rate by approx. 4.1527, 3.4243, 3.9801 and $4.0025 \%$, respectively.
\end{abstract}

\section{Introduction}

High-yielding hybrid rice breeding is one of the most important methods to increase rice production. By crossing two diverse parental lines, high-yielding hybrid varieties are produced. In a self-pollinating plant, this task is quite complex in case of rice, because it has small floral organs and low outcrossing rate. Therefore, breeding of high outcrossing rate maternal parent, and improvement of the hybrid seed production efficiency are essential for the commercialization of hybrid rice.

The factors affecting the production efficiency of hybrid seeds are very complicated. Among these factors, the stigma exsertion, as an important component for increasing pollination and seed set, are specially highlighted (Kato and Namai 1987). Thus to develop a high exserted stigmas maternal parent is expected, it is not only to help to trap more pollen dispersed from a paternal parent, but also to overcome the barrier of pollination caused by the differences in the flowering time or date between the parents.

In rice species, wild variability exists in stigma exsertion (Virmani and Athwal 1973). A total of 2065 lines of the African rice germplasm collections and breeding materials in the IITA, including 1787 Oryza sativa, 184 O. glaberrima, 66 O. barthii, 11 O. punctata, 10 O. stapfii and 7 O. longistaminata were examined to identify lines with exceptional stigma exsertion. Of these, 1 , $55,35,100,20$ and $86 \%$ were found to have a high degree of stigma exsertion respectively. The 18 varieties and lines of $O$. sativa were found to have exceptional stigma exsertion (Ying and Zhang 1989). Ling and $\mathrm{Zu}$ (1988) investigated the exserted stigma rate of 564 cultivated rice, of these, 105 varieties were 0\%, 235 varieties were between 1 and 10\%, and a high stigma exertion percentage was found in all 17 wild rice allies. Virmani and Athwal (1974) reported that the exserted stigma rate of a indica rice cultivar, Taichung No.1, was the lowest and only $0.2 \%$, while that of wild rice was as high as $91.8 \%$.

The exserted stigma rate also varies widely in rice cultivars of different types and origin. According to the investigation reported by $\mathrm{Xu}$ et al. (1986), the exserted stigma rate of indica rice was higher (19.85\% in 1983 and $19.34 \%$ in 1984) than that ( $5.91 \%$ in 1983 and $7.61 \%$ in 1984) of

*Author for correspondence: <tongjiping@ sina.com>. 
japonica rice, the exserted stigma rate of upland rice was higher (18.76\% in 1983 and $30.52 \%$ in 1984) than that (11.24\% in 1983 and $5.34 \%$ in 1984) of lowland rice and the exserted stigma rate $(25.20 \%)$ of japonica rice from Yunnan plateauwas higher than that $(3.25 \%)$ of japonica rice from Taihu valley (Xu et al. 1986).

In order to study the interaction between genotype of exserted stigma rate of rice and environment,by using the RIL population derived from XieqingzaoB/Miyang 46, Shen et al.(2006) mapped the QTLs with main effects, epistasis, and G×E interaction effects for exserted stigma rate of rice in two environments (Hainan and Hangzhou). No GxE interaction effect and no epistasis by environment effects were detected, this results of GxE interaction effects for exserted stigma rate answered a question of widespread concern, it was consistent with the cognition in rice breeding practice (Shen et al. 2006). In rice species, to be a QTLs, polygenes controls stigma exsertion (Virmani and Athwal 1973, 1974),but the character of the stigma exsertion of rice itself still inherits stably. Therefore, the high exerted stigma rate rice lines are applicable to actual breeding as an effective gene resource.

Recent rapid progress in DNA marker technology has enabled the detection of quantitative trait loci controlling complex genetic traits. To be a highly heritable trait, many QTL analysis for exserted stigma rate of rice has been conducted successfully (Li et al. 2001,Yamamoto et al. 2003, Li et al.2003, Miyata et al. 2007,Yan et al. 2009, Yue et al. 2009, Deng et al. 2010).

In order to establish a theoretical genetic basis, and to develop a high exserted stigmas Cytoplasm-nuclear Male Sterile (CMS) lines, Photosensitive Genic Male Sterility (PGMS) lines were used for actual hybrid rice breeding. By Using DNA marker technique and a recombinant inbred line population derived from an important Indica rice cross between Zhenshan97 and Minghui63, the parents of Shanyou63, the most important hybrid rice variety most-widely grown in China, a molecular marker-based analysis of quantitative trait loci (QTL) for sigma exsertion was performed in the present study.

\section{Material and Methods}

In this study, a population of 241 recombinant inbred lines (RILs) was used, the RILs were derived from a cross between Minghui 63 and Zhenshan 97. The plant materials were planted in the field during the rice-growing seasons 2009 at the experiment stations of Tianjin crop research institute at Tianjin, China. Seeds were planted in a seedbed in mid-May and were then transplanted to the field in mid-June. The planting density was $13.3 \mathrm{~cm}$ between plants in a row (the rows were $16.7 \mathrm{~cm}$ apart). Field management, including irrigation, fertilizer application and pest control, followed the normal agricultural practice.

The sigma exsertion were recorded 7-10 days after heading, five normal panicles from five representative plants were sampled from each RIL for the sigma exsertion investigation. Since there was no difference between the QTLs for single stigma exsertation and the total percentage of exserted stigma, the frequency of the exserted stigma was only defined as the total rate (\%) of the single exserted stigma and dual exserted stigma (Li et al. 2003, Deng et al. 2010).

Previously the molecular marker of 168 RFLPs and 52 SSRs were as described by Xing et al. (2002). To reduce the gaps on the map, another 34 SSR markers were added in certain regions. The SSR assay was preformed as previously described by Wu and Tanksley (1993), and the RFLP marker assay were preformed as described Liu et al. (1997), respectively. The linkage map covered a total of $1796 \mathrm{cM}$ with an average interval of about $7.1 \mathrm{cM}$ between adjacent marker loci, the linkage map consisted of 254 DNA markers. 
Molecular marker linkage maps were constructed, and QTL analysis was performed by using QTL IciMapping version 4.0.1.0 . The putative QTL for sigma exsertion was estimated with a calculated LOD score after 1,000 permutation tests (Wang et. al. 2014)).

\section{Results and Discussion}

The phenotypic values of sigma exsertion from the RIL population and their parents are presented in Table 1. In the RIL population, sigma exsertion showed a continuous variation (Fig. 1). This data suggested that the trait of sigma exsertion fitted the normal distributions and that the data for sigma exsertion rate were suitable for QTL mapping.

Table 1. Stigma Exsertion Rate of RIL Population.

\begin{tabular}{ccccccccc}
\hline ZS97B $(q)$ & MH63(ぷ) & Sample size & Mean & Variance & S.E. & Minimum & Maximum & Range \\
\hline 17.03 & 58.82 & 221 & 18.2651 & 229.6705 & 15.1549 & 0.15 & 73.29 & 73.14 \\
\hline
\end{tabular}

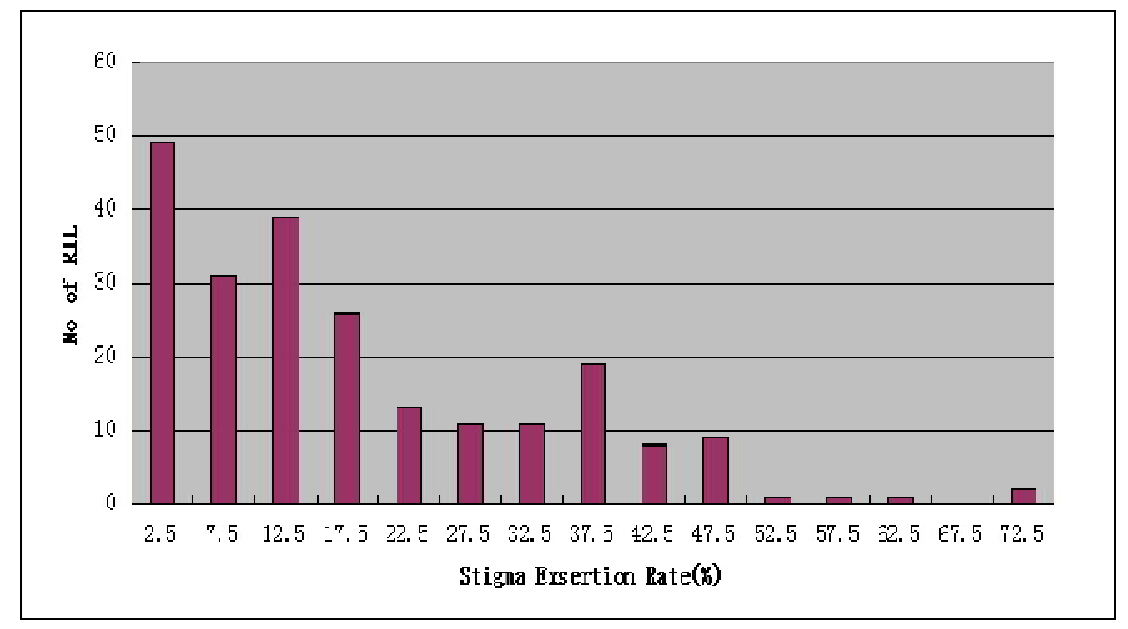

Fig. 1. Stigma Exsertion Rate (\%) Distribution of RIL.

The QTL analysis for exserted stigma rate was conducted using QTL Ici Mapping version 4.0.1.0. Six prominent QTL were detected for the exserted stigma rate on chromosome 6, 2, 9, 3, 5, and 1, respectively (Figs 2-3, Table 2). qSER-1-1 explained $8.1515 \%$ of the total phenotypic variance, and the ZS97B allele increased exserted stigma rate by about $4.3484 \%$. qSER-2-1 explained $4.6657 \%$ of the total phenotypic variance, and the ZS97B allele increased exserted stigma rate by about $3.2836 \%$. qSER-5-1 explained $7.2387 \%$ of the total phenotypic variance, and the MH63 allele increased exserted stigma rate by about $4.1527 \%$. qSER-6-1 explained $4.8997 \%$ of the total phenotypic variance, and the MH63 allele increased exserted stigma rate by about $3.4243 \%$. qSER-15-1 explained $6.925 \%$ of the total phenotypic variance, and the MH63 allele increased exserted stigma rate by about $3.9801 \%$. qSER-18-1 explained $6.9291 \%$ of the total phenotypic variance and the MH63 allele increased exserted stigma rate by about $4.0025 \%$. All the LOD score of QTLs for stigma exsertion rate (\%) are listed in Table 2. 


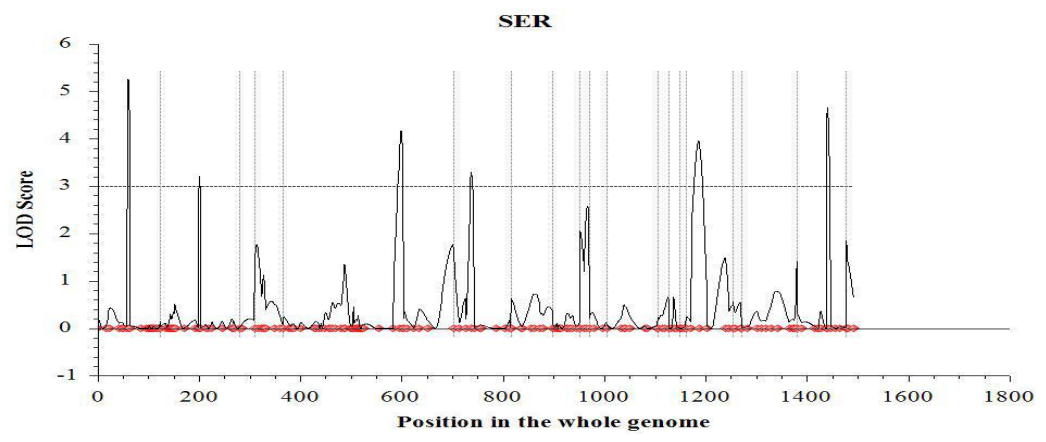

Fig. 2. QTL Distribution in the Whole Linkage Map.

Table 2. The LOD Score of QTLs for Stigma Exsertion Rate (\%).

\begin{tabular}{lclccc}
\hline QTL Name & Chromosome & Interval & LOD & PVE (\%) & Add \\
\hline qSER-1-1 & 6 & P-RZ588 & 5.2586 & 8.1515 & -4.3484 \\
qSER-2-1 & 2 & R1843-RM29 & 3.2077 & 4.6657 & -3.2836 \\
qSER-5-1 & 9 & RM215-RG667 & 4.1615 & 7.2387 & 4.1527 \\
qSER-6-1 & 3 & RZ403-C1087 & 3.2866 & 4.8997 & 3.4243 \\
qSER-15-1 & 5 & RZ649-C734b & 3.9465 & 6.925 & 3.9801 \\
qSER-18-1 & 1 & G393-R2201 & 4.6584 & 6.9291 & 4.0025 \\
\hline
\end{tabular}

Note: 1) PVE (\%): Phenotypic Variation Explained (\%); 2), Add: Additive Effect.
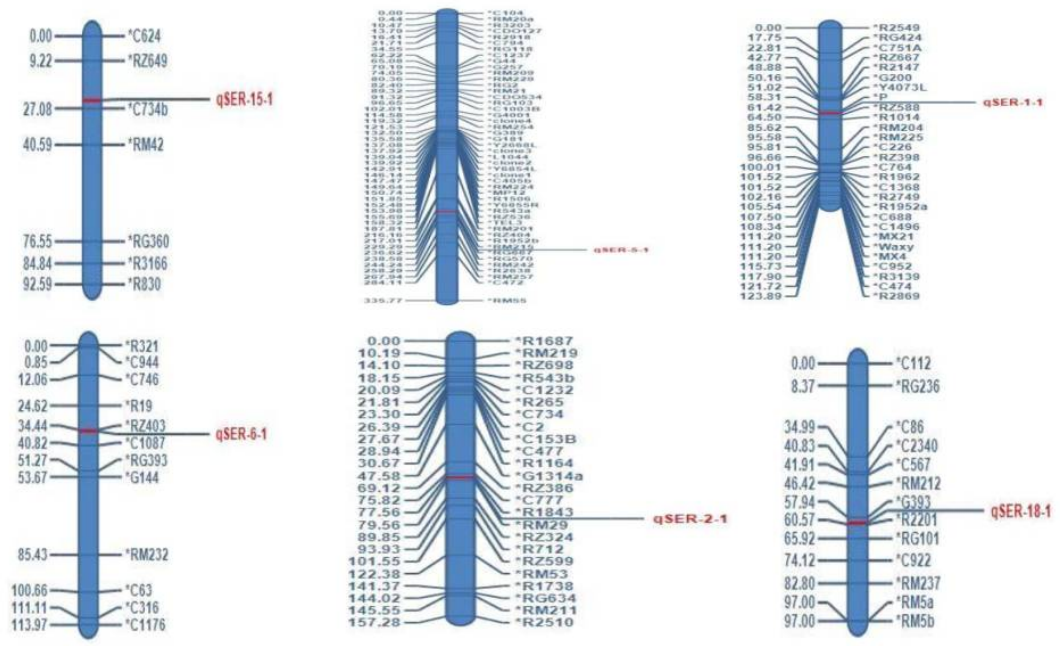

Fig. 3. QTL Map for Exserted Stigma Rate in [ZS97B/MH63] RIL Population.

In order to analyze the molecular and genetic structure underlying complex quantitative traits, quantitative trait locus mapping (QTL mapping) is a hot research topic and commonly used at present. This method has been wildly applied in crop improvement program for creating quality 
germplasm resources. In hybrid rice, stigma exsertion identified as a major component for increasing pollination and seed set is thought to be a complex quantitative traits controlled by polygenes (Virmani and Athwal 1973, 1974, Kato and Namai 1987). For many years, not only geneticists but also rice breeders have took attempts to improve the genetic characters.

By using a $\mathrm{BC}_{1}$ population derived from a crossing between common wild rice Oryza rufipogon Giff (from Dongxiang, Jiangxi Province, China) and an elite Indica Guichao 2, two QTLs controlling ratio of stigma cxsertionone were located on Chromosomc 5 at the region between R1553 and C2289 and located on Chromosome 8 at the region between R1963 and G1149 (Li et al. 2001). Using Recombinant Inbred Lines population (RILs), seven QTLs related to the rate of the exserted stigma were located on chromosomes 3, 6, 8 and 11 at the IR24 allele, respectively (Yamamoto et al. 2003). Using a population of 127 DH derived from a cross between a low-percentage of exserted stigma japonica variety Jingxi 17 and a high-percentage of exserted stigma Indica variety Zaiyeqing 8, two QTLs ( $q P E S-2, q P E S-3)$ for percentage of exserted stigma were located on chromosome 2 and 3, respectively ( $\mathrm{Li}$ et al. 2003). Shen et al. (2006) have found that one QTL $q S E 6-1$ explained $14.14 \%$ of the total phenotypic variance, $q S E 6-1$ controls the ratio of stigma cxsertion, the XQZB allele increased exserted stigma rate by about $5.10 \%$, the QTL was located on chromosome 6 (Shen et al. 2006). qES3, a highly significant QTL, explained about $32 \%$ of the total phenotypic variance, and it increased about $20 \%$ of the frequency of the exserted stigmas at the IR24 allele, $q E S 3$ was located on chromosome 3 at the centromeric region (Miyata et al. 2006). Li has reported three QTLs controlling ratio of stigma exsertion (Li 2009). Yan et al. (2009) has reported four markers associated with single stigma exsertion, six markers associated with dual exsertion and five markers associated with total exsertion. Moreover, Yue et al. (2009) has reported that QTLs affect stigma exertion rate: 1) two QTLs of percentage of dual exerted stigma were detected on chromosomes 3 and 9 with accumulated contribution of $15.98 \%, 2$ ) three QTLs of percentage of single exserted stigma were mapped on chromosomes 3, 7 and 9 with accumulated contribution of $21.44 \%$, 3) five QTLs of percentage of total exserted stigma were mapped on chromosomes 3,4,7 and 9, the total contribution rates of the five QTLs was 39.42\%.

Deng et al. (2010) has reported the QTLs mapping for percentage of exserted stigma in rice by using Nuo5/YouI $\mathrm{F}_{2}$ population, where three QTLs controlling ratio of stigma exsertion, qPES-2, qPES-5 and qSPES-8, were located on chromosome 2 at the region of RM1285-RM12595, located on chromosome 5 at the region of RM17952- RM18114, and located on chromosome 8 at the region of RM8020-RM7080 respectively. The LOD peak of the QTLs were $3.54,4.79,3.85$, respectively; the phenotypic variation was $10.1,11.1,9.0 \%$ and all their positive alleles were from YouIB. By using YZX6/II-32B RIL Population. Yin (2013) has reported 16 QTLs controlling ratio of stigma exsertion, those QTL were located on chromosome1, 3,5,6,7 and 9, respectively. Using a RIL population derived from ZX and CX29B, 11 QTLs for stigma exsertion rate were detected, two QTLs located on chromosome 1, designated qSel; and four QTLs located on chromosome 6, designated qSe6. qSel regions from ZX showed a single stigma exsertion rate increased by $16.24 \%$ and a dual stigma exsertion rate increased by $18.35 \%$; qSe6 regions from CX29B showed a single stigma exsertion rate increased by $20.47 \%$ and a dual stigma exsertion rate increased by $16.87 \%$ (Li et al. 2014).

Over recent years, numerous studies have been performed on QTL mapping of stigma exsertion rate in rice, resulting in many achievements. Different researchers, using different research materials, came up with different results, therefore, how to integrate and utilize these results, and apply it to the rice breeding practice , and how to polymerize multiple desirable alleles of stigma exsertion into the core resource by marker-assisted selection, remain important and realistic objects that should be addressed by future research. 


\section{Acknowledgements}

The authors wish to thank Dr. Guohua Yang from the Wuhan University for kindly providing the RILs population. They are also grateful to Prof. Jiankang Wang and Dr. Luyan Zhang from the Institute of Crop Science Chinese Academy of Agricultural Sciences for their assistance in using the QTL IciMapping version 4.0.1.0 software. This work was supported by the National Nature Science Foundation of China (31071396), the Key Projects of Nature Science Foundation of Tianjin Municipality (11JCZDJC17400), and the President Nature Science Foundation of the Tianjin Academy of Agricultural Science (09012).

\section{References}

Deng YD, Ying Z, Shi YY, Xiao CL and Zhang HQ 2010. Mapping of QTLs for percentage of exserted stigma in rice. J. Hunan Agricul. Univ. (Natural Sci.). 36(4): 373-376.

Kato $\mathrm{H}$ and Namai $\mathrm{H}$ 1987. Floral characteristics and environmental factors for increasing natural outcrossing rate for $\mathrm{F}_{1}$ hybrid seed production of rice Oryza sativa L. Jpn. J. Breed. 37: 318-330.

Li Ch, Sun Ch, Mu P, Chen L, Wang XK 2001. QTL analysis of anther length and ratio of stigma exsertion, two key traits of classification for cultivated rice (Oryza sativa L.) and common wild rice (O. rufipogon Griff.). Acta Genetica Sinica 28(8):746-751.

Li P B, Feng FC, Zhang L, ChaoY, Gao GJ and He YQ 2014.Genetic mapping and validation of quantitative trait loci for stigma exsertion rate in rice. Molecular Breeding 34.4: 2131-2138.

Li WH, Dong GJ, Hu XM, Teng Sh, Guo LB, Zeng DL and Qian Q 2003. QTL analysis for percentage of exserted stigma in rice (Oryza sativa L.). Acta Genetica Sinica 30(7): 637-640.

Ling ZM and Xu BG 1988. Studies on the stigma exsertion after anthesis in rice. Acta Agricalturae Universitatis Pekinensis 14(4): 388-392.

Li Y 2009. Stigma several quantitative traits in rice research and QTLs Genetic Analysis. Sicuan Normal University master's thesis.

Miyata M, Yamamoto T, Komori T and Nitta N 2007. Marker-assisted selection and evaluation of the QTL for stigma exsertion under japonica rice genetic background. Theor. Appl. Gen. 114: 539-548.

Shen SQ, Zhuang JY, Bao JS, Shu QY and Xia YW 2006. Analysis of QTLs with Main, Epistasis and G x E Interaction Effects of Stigma Extruding Trait in Rice. J. Biomathem. 21(4): 610-614.

Virman SS and Athwal DS 1973. Genetic variability in floral characters influencing outcrossing in Oryza sativa L. Crop Sci.13: 66-67.

Virmani SS and Athwal DS 1974. Inheritance of floral characters influencing outcrossing in rice. Crop Sci. 14: 350-353.

Wang JK, Li H H, Zhang LY and Meng L 2014. QTL IciMapping version 4.0.1.0.

Xu YB, Shen ZT, Yang ZN and Ying CS 1986.Study on improving percentage of cross pollination in rice I. analysis on variation of stigma exsertion in Oryza sativa L. 12(4): 359-368.

Yamamoto T, Takemori N, Sue N and Nitta N 2003. QTL analysis of stigma exsertion in rice. Rice Genet Newsl. 20: 33-34.

Yan WG, Li Y, Agrama HA, Luo DG, Gao FY, Lu XJ and Ren GJ 2009. Association mapping of stigma and spikelet characteristics in rice (Oryza sativa L.). Molecular Breeding 24(3): 277-292.

Yin Ch 2013. QTL mapping of the rate of exserted stigma and protein content in rice (Oryza sativa L.). Huazhong agricultural University. master 's thesis. 2013.

Ying CS and Zhang SQ 1989.Studies on the character of stigma exsertion among some of Oryza species. Chinese JR Sci. 3(2): 62-66.

Yue GH, Mei HW, Pan BR, Lou J, Li MS and Luo LJ 2009. Mapping of QTLs affecting stigma exsertion rate of Huhan IB as a CMS maintainer of upland hybrid rice. Acta Agricul. Zhejiangensis 21(3): 241-245. 